\title{
Nuclear physics and astrophysics of the $r$-process
}

\author{
Y.-Z. Qian ${ }^{\mathrm{a} *}$

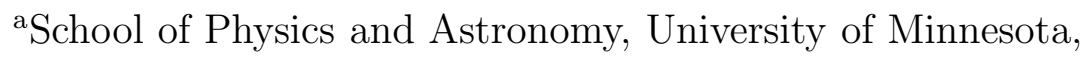 \\ Minneapolis, MN 55455, U.S.A.
}

Some nuclear and astrophysical aspects of the $r$-process are discussed. Particular attention is paid to observations of abundances in metal-poor stars and their implications for the astrophysical site and yield patterns of the $r$-process. The effects of supernova neutrinos and related nuclear processes on the yield patterns are explored. The uncertainties in the theoretical nuclear input for the $r$-process are discussed and the need for experimental data is emphasized.

\section{INTRODUCTION}

An overview of the nuclear physics and astrophysics involved in $r$-process nucleosynthesis is given here. This is largely a concise summary of a recent review on the subject [1] by this author. An earlier but still very useful review is [2].

When the universe was born 13.5 Gyr ago, the big bang imprinted the baryonic matter with a primordial composition of $76 \%$ of $\mathrm{H}$ and $24 \%$ of ${ }^{4} \mathrm{He}$ (by mass). By comparison, when the solar system was formed $4.6 \mathrm{Gyr}$ ago, its birth material contained $71 \%$ of $\mathrm{H}$, $27 \%$ of ${ }^{4} \mathrm{He}$, and $2 \%$ of heavier nuclei. This change in nuclear abundances resulted from 9 Gyr of nucleosynthesis in stars. Two neutron capture processes are especially important as they are the dominant mechanisms for producing the nuclei heavier than the Fe group. These are the slow $(s)$ neutron capture process in stars of $\sim 1-8 M_{\odot}$ and the rapid $(r)$ neutron capture process to be discussed here. Since the $s$-process operates close to the stable nuclei and its astrophysical site has been established, its contributions to the solar system abundances are relatively well understood [34]. Consequently, the $r$-process contributions can be obtained by subtracting the $s$-process contributions from the total solar abundances. The solar $r$-process (abundance) pattern derived this way is shown in Figure 1. It can be seen that the prominent features of this pattern are the peaks at mass numbers $A \sim 130$ and 195 .

The origin of these peaks can be understood as follows. At the beginning of the $r$ process, there are many neutrons and some seed nuclei to capture them. By definition, neutron capture occurs much more rapidly than $\beta$ decay during the $r$-process. So the $r$-process path moves toward the neutron-drip line as the seed nucleus keeps capturing neutrons. However, at some point, the separation energy of the next neutron to be captured becomes so small that it will be quickly disintegrated by the photons in the $r$-process environment. At this so-called waiting point, there is a tug of war between

\footnotetext{
*This work was supported in part by US DOE grants DE-FG02-87ER40328 and DE-FG02-00ER41149.
} 


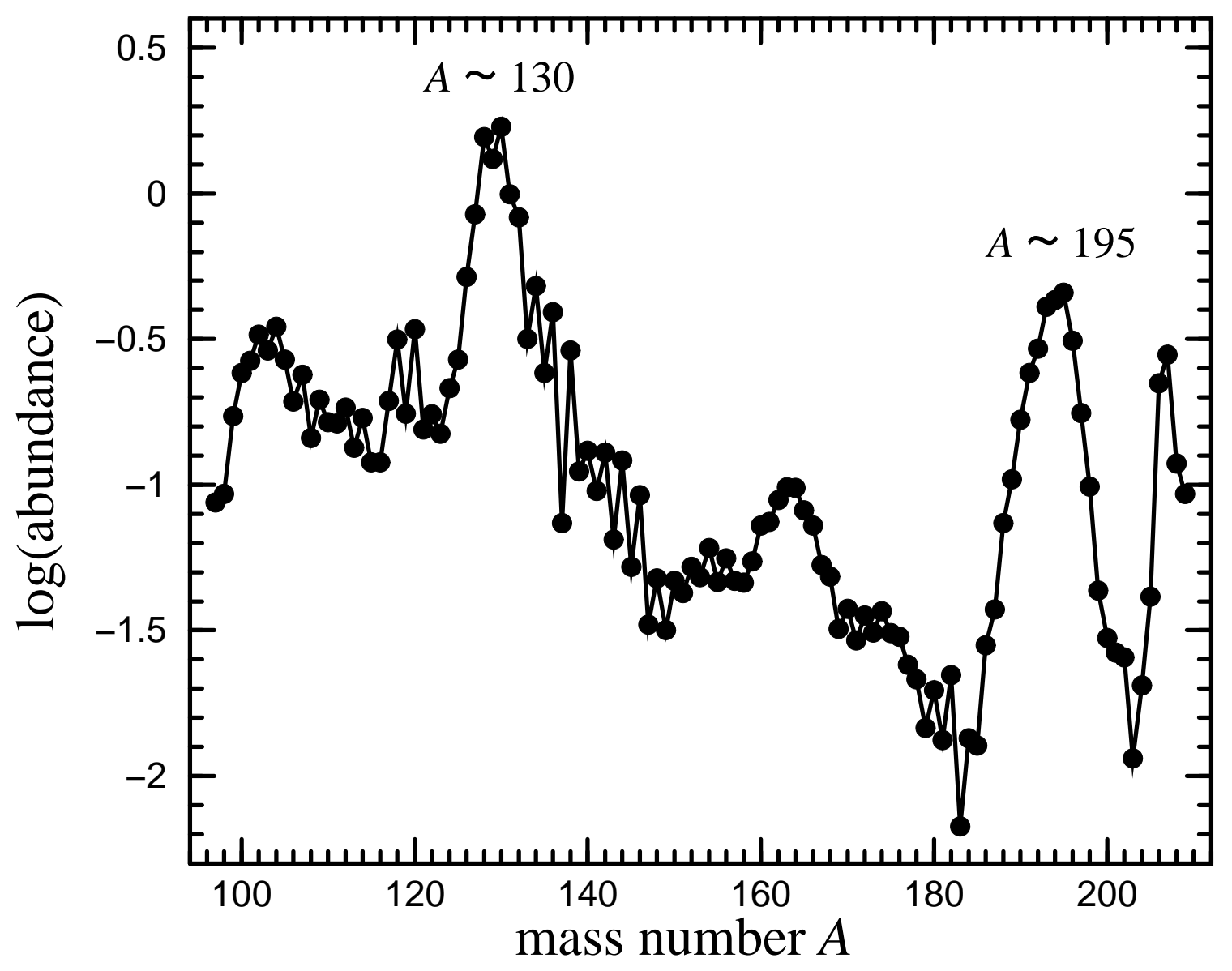

Figure 1. Solar $r$-process (abundance) pattern as derived in 3 .

neutron capture and photodisintegration. No further net neutron capture can occur until the waiting-point nucleus $\beta$-decays to a new species. The tug of war then repeats at the next waiting point. Through such a series of neutron capture and $\beta$ decay, a population of very neutron-rich progenitor nuclei far from stability are produced. Clearly, at a given proton number, the progenitor abundance is piled at the corresponding waiting-point nucleus. The more slowly this nucleus $\beta$-decays, the more abundant it will be. The extremely slow $\beta$-decay of the progenitor nuclei with magic neutron numbers $N=82$ and 126 then leads to peaks in the progenitor abundance distribution. These become the peaks at $A \sim 130$ and 195 in the solar $r$-process pattern following successive $\beta$ decay of the progenitor nuclei upon cessation of neutron capture.

\section{ASTROPHYSICAL MODELS AND OBSERVATIONS}

As discussed above, given an initial state with many neutrons and some seed nuclei, simple nuclear systematics will produce the dominant features of the solar $r$-process pattern. On the other hand, how the neutrons and seed nuclei are provided must be determined 
by the astrophysical site of the $r$-process.

One possible site is supernovae that occur when a stellar core collapses into a compact neutron star. The neutron star has an enormous gravitational binding energy of $\sim 10^{53}$ erg. Due to the high temperature and density encountered during the collapse, the most efficient way to release this energy is to emit $\nu_{e}, \bar{\nu}_{e}, \nu_{\mu}, \bar{\nu}_{\mu}, \nu_{\tau}$, and $\bar{\nu}_{\tau}$. Near the neutron star, the temperature is several $\mathrm{MeV}$ and the material is dissociated into neutrons and protons. As the neutrinos emitted from the neutron star pass through this material, some of the $\nu_{e}$ and $\bar{\nu}_{e}$ are captured by the neutrons and protons, respectively:

$$
\begin{gathered}
\nu_{e}+n \rightarrow p+e^{-}, \\
\bar{\nu}_{e}+p \rightarrow n+e^{+} .
\end{gathered}
$$

The energy deposited by these $\nu_{e}$ and $\bar{\nu}_{e}$ heats the material and drives a mass outflow usually referred to as the neutrino-driven wind [5]. The reactions in equations (11) and (2) not only provide the heating to drive the wind but also interconvert neutrons and protons, thereby determining the neutron-richness of the wind. Since the $\bar{\nu}_{e}$ producing the neutrons have a higher luminosity and a higher average energy than the $\nu_{e}$ producing the protons, the wind is neutron rich (e.g., 67]). As the wind expands away from the neutron star, its temperature and density decrease and various nuclear reactions take place to change its composition. Essentially all the protons are assembled into $\alpha$-particles when the temperature $T$ drops to $\sim 0.5 \mathrm{MeV}$. The material at this $T$ dominantly consists of neutrons and $\alpha$-particles. As $T$ drops further, an $\alpha$-process occurs to burn neutrons and $\alpha$-particles into heavier nuclei $[8$. By the time the Coulomb barrier eventually stops all charged-particle reactions at $T \sim 0.25 \mathrm{MeV}$, nuclei with $A \sim 90$ have been produced. These nuclei then become the seed nuclei to capture the remaining neutrons during the subsequent $r$-process. This is the neutrino-driven wind model of $r$-process nucleosynthesis in supernovae (e.g., 9101112 ).

Another $r$-process model takes advantage of the neutrons inside the neutron star. When an old neutron star merges with another neutron star or a black hole in a binary due to energy loss from gravitational radiation, some neutron star matter is ejected (e.g., [13 14, 15 16]). An $r$-process could occur during the decompression of this extremely neutron-rich ejecta (e.g., [17 18]).

Both the neutrino-driven wind and the neutron star merger models have merits. However, due to our insufficient understanding of supernovae and neutron star properties, it is difficult to demonstrate ab initio how a robust $r$-process occurs in either model. While the conditions required for an $r$-process have been explored to some detail (e.g., [192021]), how these conditions can be realized without parametrization in the neutrino-driven wind or neutron star mergers is still an unresolved issue (e.g., [7,18,22]).

\subsection{Supernovae vs. neutron star mergers: observational clues}

Observations of elemental abundances in old Galactic halo stars have shed important light on the astrophysical site of the $r$-process. These stars are very poor in "metals" such as Fe since they were formed very early in the Galaxy when only a small number of supernovae had occurred to provide Fe. With a rate $\sim 10^{3}$ times lower than supernovae, neutron star mergers would not have occurred at all for such early times. If neutron star mergers were the major source for $r$-process elements such as Eu, then very metal-poor 
stars would have no Eu. This is in strong conflict with the substantial Eu abundances observed in stars having Fe abundances as low as $\sim 10^{-3}$ times solar and also with the substantial Ba abundances (at such low metallicities corresponding to early times, only the $r$-process associated with fast-evolving massive progenitors can contribute to the Ba in the interstellar medium) observed in stars having $\mathrm{Fe}$ abundances as low as $\sim 10^{-4}$ times solar (e.g., 2324 25]). These observations cannot be accounted for by neutron star mergers being the major $r$-process site unless the rate of these events is close to that of supernovae. Such a high neutron star merger rate is very unlikely and it is much more probable that supernovae are responsible for both the $r$-process and Fe enrichment of metal-poor stars (e.g., 26 27]). In the rest of the discussion, it will be assumed that supernovae are the major $r$-process site.

\subsection{Diverse supernova $r$-process sources: more observational clues}

Different conditions are required to produce the peaks at $A \sim 130$ and 195, respectively, in the solar $r$-process pattern. These peaks may be produced by the different kinds of supernovae associated with the neutrino-driven wind model of the $r$-process. The diversity of supernova sources for the $r$-process was first suggested in 28] based on meteoritic data and later received support from observations of the abundances for a wide range of $r$-process elements in the metal-poor star CS 22892-052 [29]. Further observations of abundances in metal-poor stars have also provided important clues in this regard.

The data on three metal-poor stars CS 31082-001 [30], HD 115444, and HD 122563 31] are shown in Figure 2. The curves to the right of the vertical line in Figure 2b represent the solar $r$-process pattern translated for comparison with the data on each star. It can be seen that for all three stars, Ba and heavier elements with $A>130$ (including the $A \sim 195$ peak at $\mathrm{Pt}$ ) closely follow the solar $r$-process pattern. Note that the absolute abundances of these elements $(\log \epsilon$ is the logarithm of the absolute abundance in appropriate units) differ by a factor of $~ 100$ for CS 31082-001 and HD 122563. In other words, if HD 122563 received contributions from one supernovae, then CS 31082-001 received contributions from $\sim 100$ supernovae. However, as shown in Figure $2 \mathrm{a}$, the abundances of $\mathrm{O}$ to Ge including Fe are essentially the same for these stars. This suggests that supernovae producing the heavy $r$-process elements with $A>130$ cannot produce any of the elements from O to Ge (e.g., 32). Since the latter elements are produced by hydrostatic and explosive burning in the shells outside the core, to avoid their production requires the supernovae to have very thin or no shells at all. These supernovae are associated with progenitors of $\sim 8-10 M_{\odot}$ 33.34 and with accretioninduced collapse of white dwarfs [35]. On the other hand, supernovae with progenitors of $>10 M_{\odot}$ produce the elements from $\mathrm{O}$ to Ge including Fe (e.g., [36]). They must then be responsible for the light $r$-process elements up to the $A \sim 130$ peak.

\section{NUCLEAR PHYSICS AND YIELD PATTERNS}

As discussed in the introduction, the peaks at $A \sim 130$ and 195 in the solar $r$-process pattern are due to the nuclear systematics of the $N=82$ and 126 closed neutron shells, respectively. The possible connections between nuclear systematics and features of the $r$ process pattern observed in metal-poor stars are discussed below. In particular, the effects of reactions induced by supernova neutrinos are explored considering that the $r$-process 

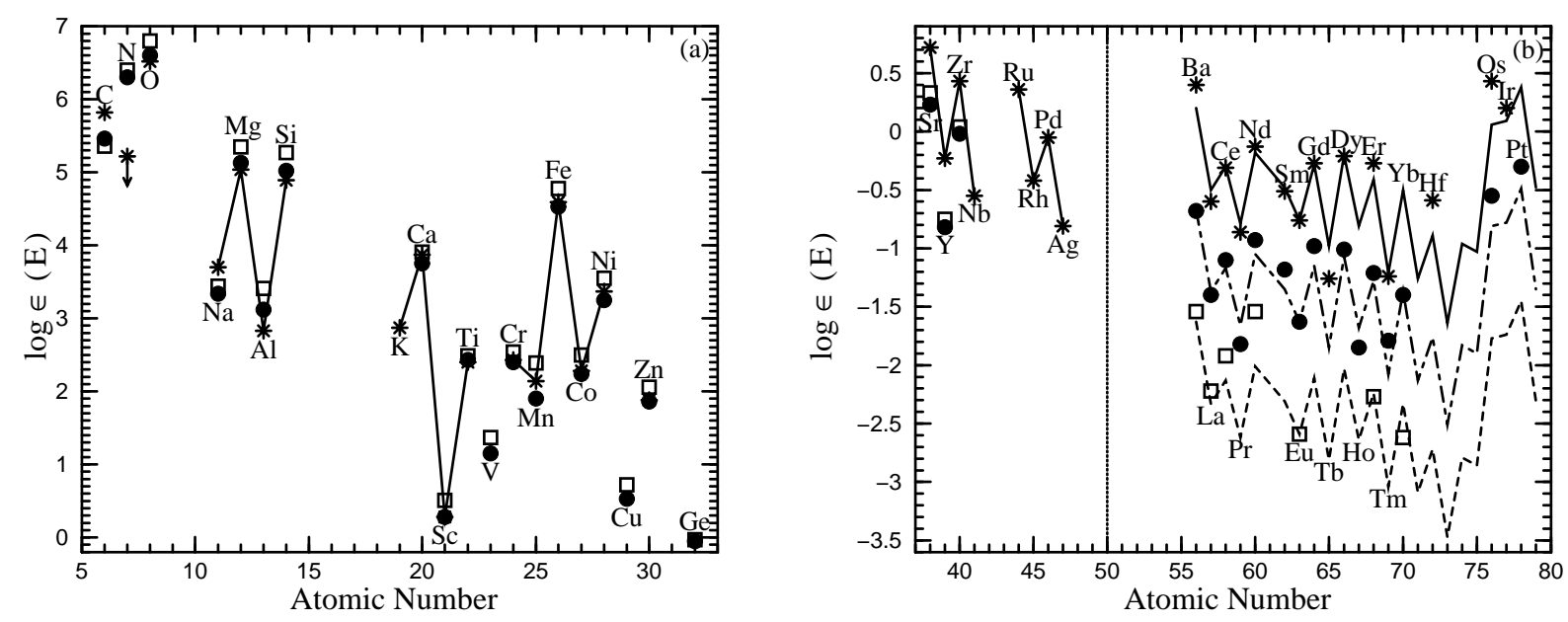

Figure 2. Comparison of the observed abundances in CS 31082-001 (asterisks: 30]), HD 115444 (filled circles), and HD 122563 (squares: [31]). (a) The data on CS 31082-001 are connected with solid line segments as a guide. Missing segments mean incomplete data. The downward arrow at the asterisk for $\mathrm{N}$ indicates an upper limit. Note that the abundances of the elements from $\mathrm{O}$ to Ge are almost indistinguishable for the three stars. (b) The data on CS 31082-001 to the left of the vertical line are again connected with solid line segments as a guide. In the region to the right of the vertical line, the solid, dot-dashed, and dashed curves are the solar $r$-process pattern translated to pass through the Eu data for CS 31082-001, HD 115444, and HD 122563, respectively. Note the close description of the data by these curves. The shift between the solid and the dashed curves corresponds to a change by a factor of $\sim 100$ in the absolute abundances.

occurs in the neutrino-driven wind. An example is given to illustrate the uncertainties in the theoretical nuclear input for the $r$-process and to highlight the need for experimental data.

\subsection{Fission and neutrino-induced reactions}

Observations have shown that $\mathrm{Ba}$ and heavier elements with $A>130$ in a number of metal-poor stars closely follow the solar $r$-process pattern (e.g., Fig. 2b). This highly regular pattern may be produced by some special nuclear process. For example, if the $r$-process occurs in an extremely neutron-rich environment, the heaviest nucleus produced may fission. The fission fragments then become the new seed nuclei to capture neutrons. This results in a cyclic flow between the fissioning nucleus and its fragments. It was shown that for certain conditions, this fission cycling can produce a solar $r$-process pattern involving only the nuclei with $A>130$ (e.g., [18]).

However, observations suggest that the actual production of the heavy $r$-process elements with $A>130$ is quite complex. The data on the metal-poor stars CS 22892-052 [29] and CS 31082-001 30] are shown in Figure 3. It can be seen that the heavy $r$-process elements are not produced alone, but along with some light $r$-process elements such as Sr, Y, and Zr with $A<130$. Further, while the abundances of the elements below $\mathrm{Pb}$ are 


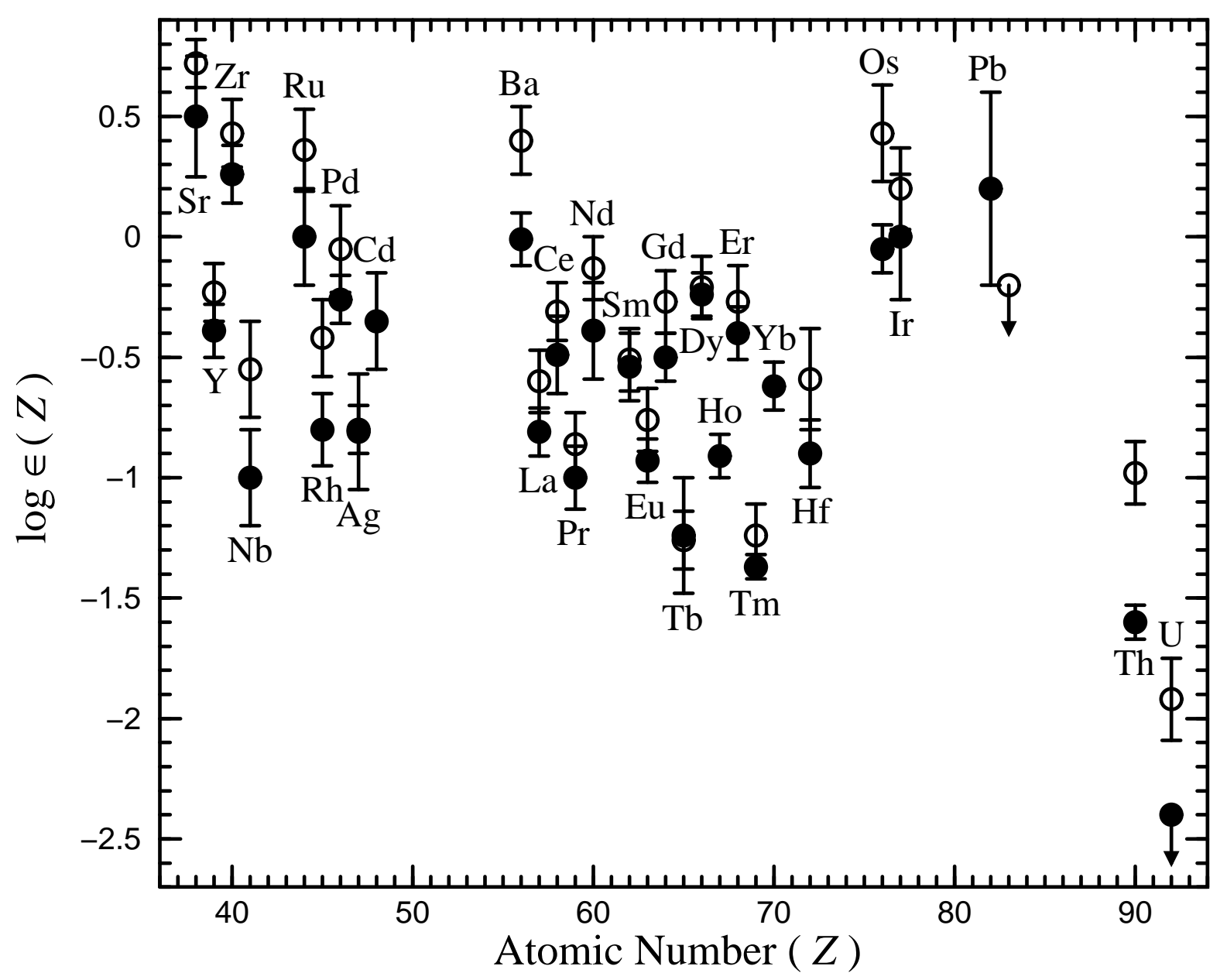

Figure 3. Comparison of the observed abundances in CS 22892-052 (filled circles: 29]) and CS 31082-001 (open circles: [30]). The open circle for $\mathrm{Pb}$ is shifted slightly for clarity. Downward arrows indicate upper limits.

essentially identical for the two stars, their Th and $U$ abundances differ greatly. These two features are in contradiction with the commonly cited virtues of fission cycling: a robust yield pattern at $A>130$ and no production of the nuclei with $A<130$ (e.g., [18]).

On the other hand, fission cycling may not occur during the $r$-process. Instead, the progenitor nuclei may fission during decay toward stability. If the $r$-process initially produces a progenitor pattern covering nuclei with $A \gtrsim 190$ with a peak at $A \sim 195$, both the light $r$-process elements with $A<130$ and the heavy ones with $130<A<190$ can be produced by fission of the progenitor nuclei during decay [37. The fission probability can be significantly enhanced by the interaction between supernova neutrinos and the progenitor nuclei 3738 . This is because $\nu_{e}$ with an average energy of $\sim 10 \mathrm{MeV}$ can excite these nuclei to $\sim 20 \mathrm{MeV}$ above the ground state through charged-current interaction while $\nu_{\mu}, \bar{\nu}_{\mu}, \nu_{\tau}$, and $\bar{\nu}_{\tau}$ with an average energy of $\sim 20 \mathrm{MeV}$ can provide similar excitation through neutral-current interaction. Of course, the extremely neutron-rich progenitor 
nuclei can also deexcite through neutron emission. It was shown that the solar $r$-process abundances at $A=183-187$ can be completely accounted for by neutrino-induced neutron emission from the progenitor nuclei in the $A \sim 195$ peak (see Fig. 4) 39 40]. Interestingly, the level of neutrino interaction required to accomplish this will also induce significant fission [37].

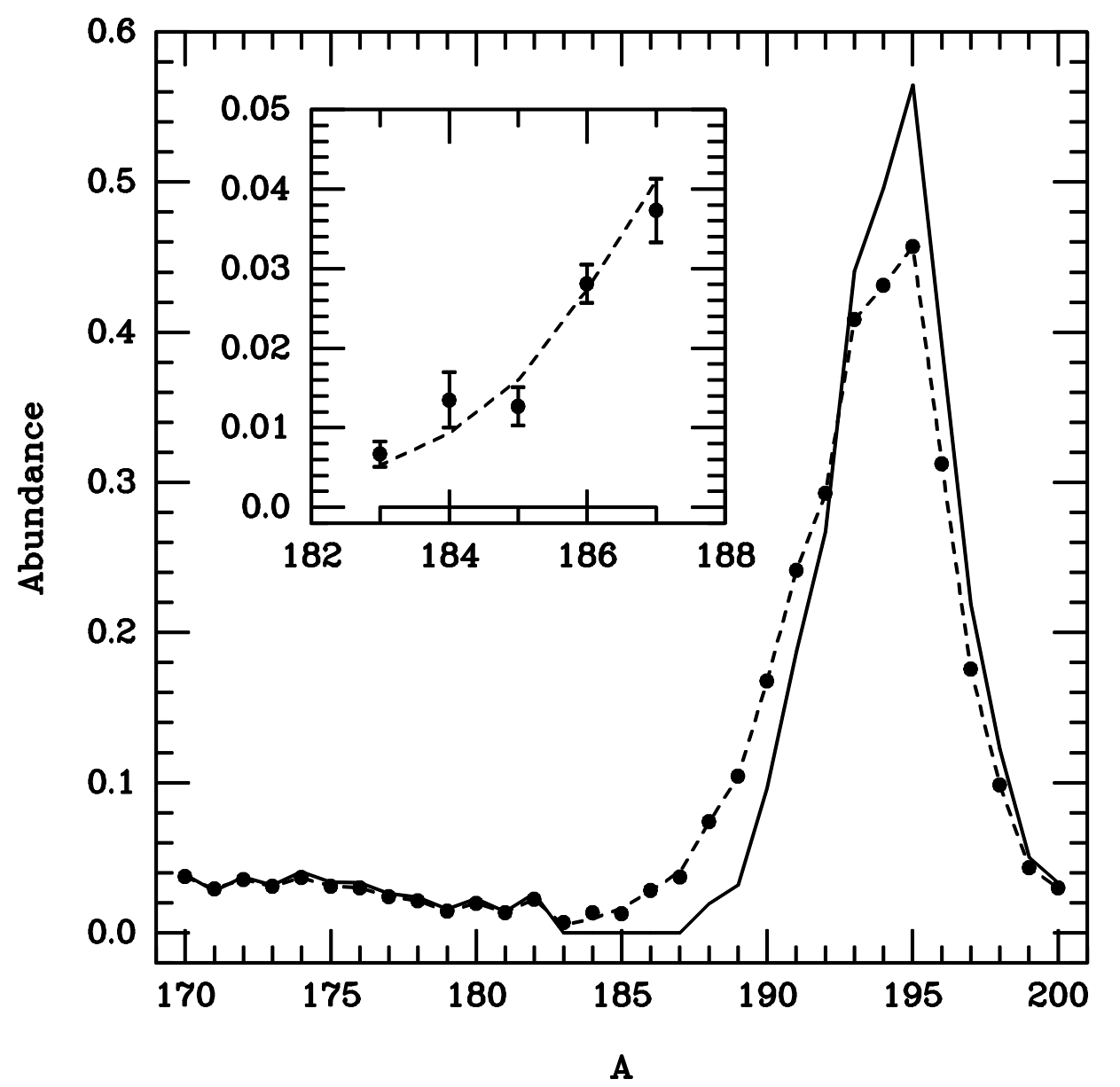

Figure 4. Production of nuclei by neutrino-induced neutron emission. The abundances before and after neutrino processing are given by the solid and dashed curves, respectively. The filled circles (some with error bars) give the solar $r$-process abundances.

\subsection{More details of yield patterns and need for nuclear data}

As can be seen from the above discussion, to understand the details of the $r$-process patterns requires a great deal of nuclear input regarding processes such as fission and neutrino interaction. Unfortunately, the majority of this input has to depend on theoretical calculations. The danger of this dependence and the importance of experimental data are illustrated below with an example concerning neutron separation energies of neutron-rich 
nuclei far from stability.

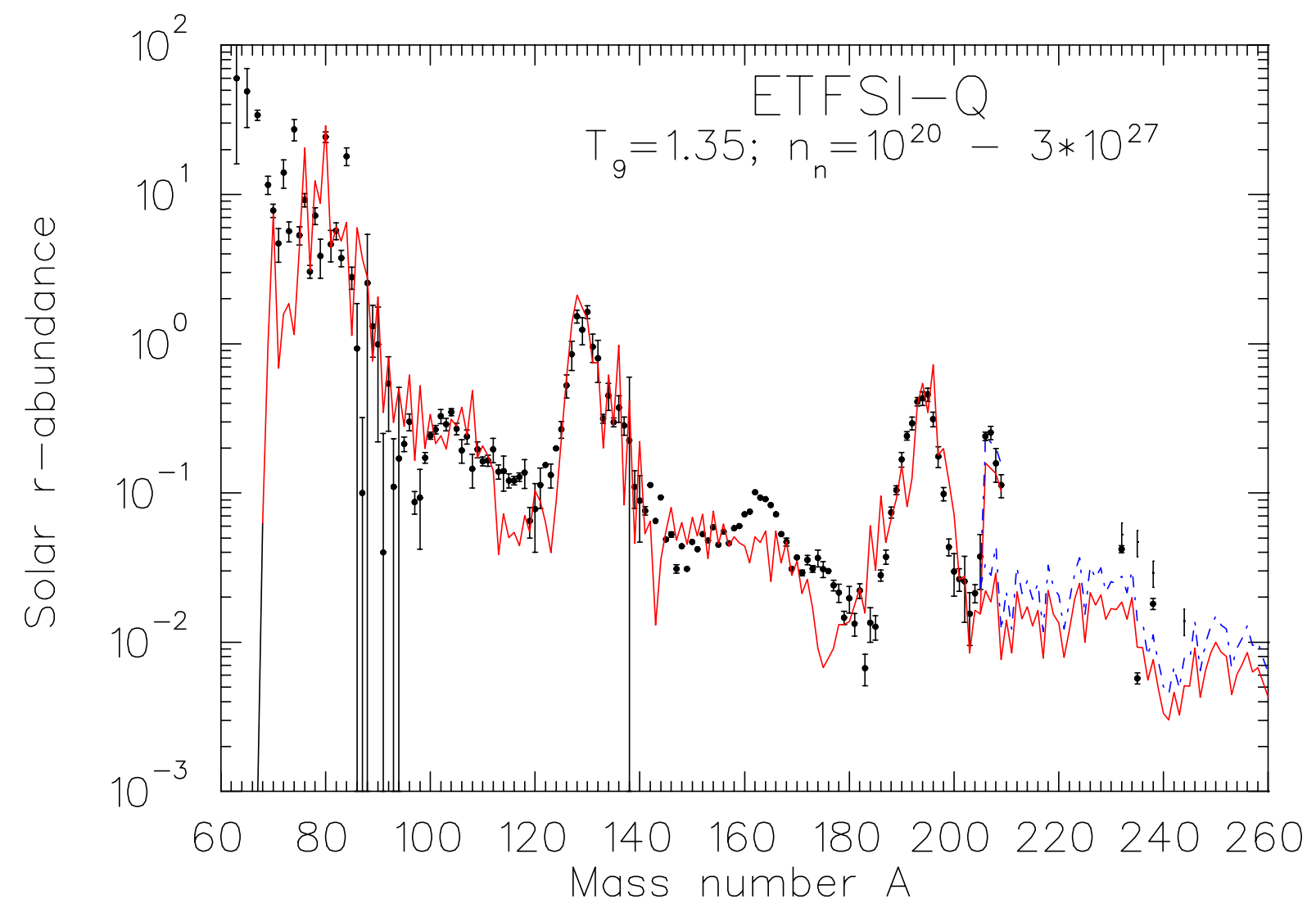

Figure 5. The solid curve represents the $r$-process pattern calculated in [41] (the dashed curve has a slightly different weight for $A>206$ ). Compared with the solar $r$-process abundances (small filled circles with error bars), the calculated pattern has severe deficiencies below the $A \sim 130$ and 195 peaks.

The detailed $r$-process pattern calculated in [41] is shown in Figure 4 along with the solar $r$-process pattern. It can be seen that the calculated pattern, among other things, greatly underproduces the nuclei below the $A \sim 130$ and 195 peaks. These deficiencies can be traced to the lack of waiting-point nuclei in the corresponding mass regions. Due to the tug of war between neutron capture and photodisintegration, the waiting-point nuclei have approximately the same neutron separation energy. In general, at a given neutron number, the neutron separation energy increases with increasing proton number and at a given proton number it decreases with increasing neutron number. So a nucleus with proton number $Z+1$ must have a larger neutron number than a nucleus with proton number $Z$ in order to have the same neutron separation energy. Typical mass formulae used in $r$-process calculations predict that for a given proton number the neutron separation energy decreases very slowly as the neutron number increases toward a magic number. Consequently, below a closed neutron shell, two nuclei with neighboring proton 
numbers must have a large difference in neutron number in order to have the same neutron separation energy. This results in a large mass gap between two neighboring waiting-point nuclei below the closed neutron shell and causes the severe underproduction below the corresponding abundance peak. It was shown that the deficiencies discussed above can be alleviated by quenching the strength of closed neutron shells in the mass formulae (e.g., 42]). Clearly, to fully resolve these deficiencies requires mass measurements in the relevant regions.

\section{CONCLUSIONS}

In summary, observations of abundances in metal-poor stars suggest that supernovae are the major $r$-process site and there are two different kinds of $r$-process. Supernovae associated with progenitors of $\sim 8-10 M_{\odot}$ and accretion-induced collapse of white dwarfs are mainly responsible for the heavy $r$-process elements with $A>130$ while supernovae with progenitors of $>10 M_{\odot}$ are mainly responsible for the light $r$-process elements up to the $A \sim 130$ peak. How the $r$-process conditions are obtained in supernovae is still

quite uncertain. As mentioned above, the neutron-richness of the neutrino-driven wind is determined by neutrino emission from the neutron star. Future studies should investigate whether neutron stars evolved from different presupernova cores have very different neutrino emission characteristics. The effects of neutrino oscillations should also be considered (e.g., [6]). Of course, magnetic fields and rotation, which are the usual "villains" in astrophysical problems, may be important, too. Further, the $r$-process yield patterns depend on a great deal of nuclear input regarding for example, masses, fission, and neutrino responses of neutron-rich nuclei far from stability. There are large uncertainties in the current theoretical nuclear input for the $r$-process calculations. Therefore, future measurements are essential in providing either direct information or guidance to theory. Hopefully, the next generation of rare isotope accelerator facilities can help put the understanding of $r$-process production on a solid basis.

\section{REFERENCES}

1. Y.-Z. Qian, Prog. Part. Nucl. Phys. 50 (2003) 153.

2. J. J. Cowan, F.-K. Thielemann, and J. W. Truran, Phys. Rep. 208 (1991) 267.

3. F. Käppeler, H. Beer, and K. Wisshak, Rep. Prog. Phys. 52 (1989) 945.

4. C. Arlandini et al., Astrophys. J. 525 (1999) 886.

5. R. C. Duncan, S. L. Shapiro, and I. Wasserman, Astrophys. J. 309 (1986) 141.

6. Y.-Z. Qian et al., Phys. Rev. Lett. 71 (1993) 1965.

7. Y.-Z. Qian and S. E. Woosley, Astrophys. J. 471 (1996) 331.

8. S. E. Woosley and R. D. Hoffman, Astrophys. J. 395 (1992) 202.

9. S. E. Woosley and E. Baron, Astrophys. J. 391 (1992) 228.

10. B. S. Meyer et al., Astrophys. J. 399 (1992) 656.

11. K. Takahashi, J. Witti, and H.-T. Janka, Astron. Astrophys. 286 (1994) 857.

12. S. E. Woosley et al., Astrophys. J. 433 (1994) 229.

13. J. M. Lattimer and D. N. Schramm, Astrophys. J. 192 (1974) L145.

14. J. M. Lattimer and D. N. Schramm, Astrophys. J. 210 (1976) 549.

15. M. Ruffert et al., Astron. Astrophys 319 (1997) 122. 
16. S. Rosswog et al., Astron. Astrophys 341 (1999) 499.

17. B. S. Meyer, Astrophys. J. 343 (1989) 254.

18. C. Freiburghaus, S. Rosswog, and F.-K. Thielemann, Astrophys. J. 525 (1999) L121.

19. B. S. Meyer and J. S. Brown, Astrophys. J. Suppl. Ser. 112 (1997) 199.

20. R. D. Hoffman, S. E. Woosley, and Y.-Z. Qian, Astrophys. J. 482 (1997) 951.

21. C. Freiburghaus et al., Astrophys. J. 516 (1999) 381.

22. T. A. Thompson, A. Burrows, and B. S. Meyer, Astrophys. J. 562 (2001) 887.

23. A. McWilliam et al., Astron. J. 109 (1995) 2757.

24. D. L. Burris et al., Astrophys. J. 544 (2000) 302.

25. J. A. Johnson and M. Bolte, Astrophys. J. 554 (2000) 888.

26. Y.-Z. Qian, Astrophys. J. 534 (2000) L67.

27. D. Argast et al., Astron. Astrophys. 416 (2004) 997.

28. G. J. Wasserburg, M. Busso, and R. Gallino, Astrophys. J. 466 (1996) L109.

29. C. Sneden et al., Astrophys. J. 533 (2000) L139.

30. V. Hill et al., Astron. Astrophys. 387 (2002) 560.

31. J. Westin et al., Astrophys. J. 530 (2000) 783.

32. Y.-Z. Qian and G. J. Wasserburg, Astrophys. J. 567 (2002) 515.

33. K. Nomoto, Astrophys. J. 277 (1984) 791.

34. K. Nomoto, Astrophys. J. 322 (1987) 206.

35. K. Nomoto and Y. Kondo, Astrophys. J. 367 (1991) L19.

36. S. E. Woosley and T. A. Weaver, Astrophys. J. Suppl. Ser. 101 (1995) 181.

37. Y.-Z. Qian, Astrophys. J. 569 (2002) L103.

38. E. Kolbe, K. Langanke, and G. M. Fuller, Phys. Rev. Lett. 92 (2004) 111101.

39. Y.-Z. Qian et al., Phys. Rev. C 55 (1997) 1532.

40. W. C. Haxton et al., Phys. Rev. Lett. 78 (1997) 2694.

41. J. J. Cowan et al., Astrophys. J. 521 (1999) 194.

42. B. Chen et al., Phys. Lett. B 355 (1995) 37. 